\title{
Clase invertida para la formación inicial de educadoras diferenciales sobre aprendizaje matemático
}

\author{
Laura Marjorie Espinoza Pastén \\ Orcid: 0000-0002-0947-0039 \\ laura.espinozap@userena.cl \\ Instituto Multidisciplinario de Ciencia y Tecnología, \\ Universidad de La Serena
}

\section{Resumen}

Actualmente, en la formación inicial de profesores algunas asignaturas de los primeros años del itinerario formativo comprenden conocimientos teóricos y abstractos, que consideran constructos y vocabulario complejo para el estudiantado. Este capítulo tiene como propósito presentar la sistematización de una innovación pedagógica efectuada en la asignatura Desarrollo y Metodología de la Enseñanza de las Matemáticas con estudiantes que cursaron cuarto semestre en la carrera de Pedagogía en Educación Diferencial, de la Universidad de La Serena. La innovación se efectuó durante la primera unidad temática de la asignatura, se diseñó según el modelo pedagógico de flipped classroom y se concretó en las sesiones de preclase y clase sugeridas por la literatura, durante cinco semanas. Además, se consideró el uso de recursos digitales y plataformas para optimizar el trabajo y los tiempos. A partir del diseño didáctico elaborado, la ejecución y valoración de la innovación pedagógica realizada, se concluye que este modelo pedagógico facilita el aprendizaje de contenidos complejos de tipo teórico y abstracto. Además, en el espacio de trabajo personal o preclase fue trascendental el compromiso de las estudiantes y la ejecución de las actividades para posteriormente llevar a cabo la clase presencial. Por último, las estudiantes que participaron de forma sistemática bajo este modelo pedagógico obtuvieron un mejor rendimiento que aquellas estudiantes que no participaron de forma activa o permanente. Como proyección de la experiencia, la clase invertida es pertinente para ser empleada en otras carreras 
de pregrado, así como en la formación técnico-profesional de nivel superior.

Palabras clave: aprendizaje matemático, clase invertida, diseño didáctico, formación inicial de profesores, innovación con uso de tecnología.

\section{Abstract}

Currently in the initial teacher training, some subjects of the first years of the training itinerary include theoretical and abstract knowledge, which consider constructs and complex vocabulary for the student body. The purpose of this chapter is to present the systematization of a pedagogical innovation carried out in the subject Development and Methodology of the Teaching of Mathematics, with students who studied the fourth semester in the career of Pedagogy in Special Education, of the University of La Serena. The innovation was carried out during the first thematic unit of the subject, being designed under the pedagogical model of flipped classroom, and materialized in the sessions of pre class and class suggested by the literature, during five weeks. In addition, the use of digital resources and platforms was considered to optimize work and times. From the elaborated didactic design, the execution and evaluation of the pedagogical innovation carried out, it is concluded that this pedagogical model facilitates the learning of complex theoretical and abstract contents. In addition, in the personal or pre-class work space, the commitment of the students and the execution of the activities was paramount to later carry out the face-to-face class. Finally, the students who participated systematically under this pedagogical model obtained a better performance than those students who did not participate actively or permanently. As a projection of the experience, the flipped classroom is relevant to be used in other undergraduate courses, as well as in higher level professional technical training.

Keywords: mathematical learning, flipped classroom, didactic design, initial teacher training, innovation with the use of technology.

\section{Problema y justificación}

En la actual formación de estudiantes de nivel superior, se busca desarrollar en ellos habilidades y conocimientos que generen autonomía para la futura labor profesional. Los actuales contextos laborales demandan de los futuros profesionales la autorregulación, la resolución de problemas, la organización, las habilidades para el trabajo en equipo y la aplicación adecuada 
de sus conocimientos a situaciones específicas laborales. Por otro lado, es importante considerar como problema el hecho de que muchas veces los contenidos que los estudiantes deben tratar en la educación superior son complejos, abstractos, de gran volumen y se requiere una adecuada gestión de toda esa información.

En este marco, una de las metodologías o modelo pedagógico de aprendizaje activo posible de usar es la clase invertida o flipped classroom, donde se re organiza la gestión de los tiempos, se prioriza el aprendizaje autónomo y participativo de los estudiantes, además de demandar habilidades de trabajo con otras personas. El modelo ha sido probado en formación de pregrado en las áreas de ingeniería, salud e idiomas, donde se ha determinado su efectividad.

Este capítulo de libro tiene como propósito presentar el diseño didáctico sistematizado de clase invertida, que fue empleado en la primera unidad de una asignatura del itinerario formativo de la carrera de Pedagogía en Educación Diferencial, de la Universidad de La Serena. Del mismo modo, ofrecer una valoración de la innovación didáctica implementada considerando el rendimiento de las estudiantes y los resultados de la autoevaluación cumplimentada por ellas. Ello podría dar luces para una posterior réplica del diseño didáctico en otros contextos, tales como la formación en otras carreras de pedagogías como en la formación técnico-profesional de nivel superior.

\section{Marco teórico}

La flipped classroom o clase invertida es un modelo pedagógico, más que una metodología de enseñanza aprendizaje, y es uno de los más utilizados como herramienta didáctica en el siglo XXI. Su diseño redistribuye los tiempos destinados a la enseñanza y el aprendizaje, dado que consta de dos partes. Primeramente, la preclase o sesión autónoma, y posteriormente, la clase (Mok, 2014). La preclase implica que se utilicen recursos digitales de aprendizaje (Blair, Maharaj y Primus, 2015), pero de manera óptima, sobre todo en educación técnica (Deyasi, Bhattacharyya, Debnath, Mukherjee y Kumar, 2019). Además, deben ser de fácil acceso y ocupar un tiempo prudente del trabajo personal del es- 
tudiante. De esta manera, la clase presencial se reservaría para el trabajo activo de los estudiantes, usualmente en equipos o de forma social, lo que suele colaborar en la satisfacción de las necesidades de los estudiantes situados en el contexto actual (Johnson y Johnson, 2014). Asimismo, la clase presencial demanda habilidades de pensamiento más complejas, tales como: analizar, evaluar, elaborar propuestas o soluciones, aplicar conocimientos de manera contextualizada, resolver situaciones, tomar decisiones, entre otras.

La clase invertida se ha implementado en el área de ciencias exactas, así como humanidades y ciencias sociales (Basal, 2015; Menegaz, Dias, Trindade, Leal y Martins, 2018; Peterson, 2016), mostrando que es efectiva cuando es implementada, independiente de la naturaleza de la carrera de educación superior o de la asignatura. Independiente del área en que se implemente, el rol docente en la clase invertida es más bien de mediador del aprendizaje (Jensen, Kummer y Godoy, 2014), dejando de lado las clases expositivas donde el estudiante solía tener un rol de escucha en la cátedra que era dictada.

Algunas publicaciones sobre clase invertida señalan que su implementación conlleva una mejora en el rendimiento de los estudiantes, así como una autoevaluación positiva de los aprendizajes alcanzados (Kong, 2014). Aunque es importante señalar, que se deben considerar para la fase de clase otros métodos activos de aprendizaje o actividades que permitan el aprendizaje autónomo del aprendiz (Jensen et al., 2014).

\section{Proceso de la innovación}

\subsection{Contexto}

La innovación didáctica se realizó con 36 estudiantes mujeres que cursaron cuarto semestre en la carrera de Pedagogía en Educación Diferencial, en la Universidad de La Serena. Específicamente, en la asignatura Desarrollo y Metodología de la Enseñanza de las Matemáticas. La asignatura es obligatoria y de carácter teórico, semestral. Además, forma parte del área de formación de la especialidad en el itinerario formativo de esta carrera pedagógica. 
Dentro de la asignatura, la innovación se efectuó en la unidad 1 de la asignatura Fundamentos Teóricos del Pensamiento Matemático. En esta unidad, se considera como resultado de aprendizaje que las estudiantes comprendan las bases teóricas clásicas y más recientes sobre la estructura psicológica y neurocognitiva evolutiva del pensamiento y aprendizaje matemático como base para el posterior diseño de intervenciones psicopedagógicas pertinentes. Por tanto, el trabajo en esta unidad implica que las estudiantes revisen, conozcan y comprendan literatura al respecto, para lo cual es necesario que posean ciertos conocimientos previos y habilidades para la comprensión y gestión de la información.

\subsection{Diseño didáctico}

Se consideró el tiempo disponible de ejecución de la unidad 1 de la asignatura, el cual fue de cinco semanas. Durante este tiempo, se ejecutaron 16 clases presenciales, distribuidas en tres clases por semana de dos horas pedagógicas cada una. Entre ellas, se efectuaron, además, clases de: presentación del programa de la asignatura y activación de conocimientos previos (n. $\left.{ }^{\circ} 1\right)$, clase preparatoria para la evaluación final de la unidad (n. ${ }^{\circ} 14$ ), ejecución de evaluación final (n. $\left.{ }^{\circ} 15\right)$ y retroalimentación de la evaluación (n. ${ }^{\circ}$ 16). Por lo tanto, el modelo pedagógico de clase invertida se aplicó a lo largo de 12 clases presenciales y sus respectivas preclases.

La secuencia didáctica efectuada durante la unidad consideró las etapas de clase invertida de Mok (2014), replicadas en Espinoza y Araya (2019):

1. Sesión autónoma: se efectuó en el tiempo personal, previa sesión presencial. Se entregaron recursos de aprendizaje por medio de plataforma Moodle: artículos, capítulos de libros, archivos de PowerPoint, guías con preguntas sobre el texto, fichas de resúmenes, cuadros comparativos, entre otros, con al menos 5 días de antelación. Se respondieron dudas por medio de correo institucional y tutorías. La sesión autónoma demandó efectuar las actividades de manera grupal, con el objetivo de aclarar dudas o buscar información adicional para facilitar la comprensión y gestión de la información. Los recursos de 


\section{aprendizaje y la actividad pedagógica vinculada se desarrolla- ron de forma parcelada, de modo que el quehacer está distri- buido, y cada recurso con su actividad de aprendizaje, en dos sesiones autónomas y dos preclases, dada su extensión. Los recursos empleados se indican en la tabla 1.}

Tabla 1. Recursos de aprendizaje en formato digital empleados durante la unidad didáctica.

\begin{tabular}{|c|c|c|}
\hline Tipo de recurso & Descripción del recurso & $\begin{array}{l}\text { Actividad de aprendizaje a } \\
\text { desarrollar en preclase }\end{array}$ \\
\hline $\begin{array}{l}\text { Artículo de revi- } \\
\text { sión teórica sobre } \\
\text { pensamiento } \\
\text { matemático. }\end{array}$ & $\begin{array}{l}\text { Bosch, M. (2012). "Apuntes teóri- } \\
\text { cos sobre el pensamiento matemá- } \\
\text { tico y multiplicativo en los prime- } \\
\text { ros niveles». Educación matemáti- } \\
\text { ca en la infancia, 1(1): 15-37. }\end{array}$ & $\begin{array}{l}\text { Guía con preguntas que impli- } \\
\text { caban componer definiciones a } \\
\text { partir del documento, síntesis de } \\
\text { información, explicación y ejem- } \\
\text { plificación de conceptos. }\end{array}$ \\
\hline \multirow[t]{2}{*}{$\begin{array}{l}\text { Capitulos de li- } \\
\text { bros sobre pensa- } \\
\text { miento métrico. }\end{array}$} & $\begin{array}{l}\text { Gómezescobar, A. y Fernández, } \\
\text { R. (2016). "Metodologías en las } \\
\text { enseñanzas de las magnitudes y } \\
\text { la medida en educación: La lon- } \\
\text { gitud". En: Ramiro-Sánchez, T.; } \\
\text { Ramiro, M. (coords.). Avances en } \\
\text { las Ciencias de la Educación y del } \\
\text { Desarrollo (pp. 594-599). Grana- } \\
\text { da: Asociación Española de Psico- } \\
\text { logía Conductual. }\end{array}$ & $\begin{array}{l}\text { Guía con preguntas de respuesta } \\
\text { de desarrollo, que implicaban re- } \\
\text { lacionar conceptos, dar ejemplos } \\
\text { contextualizados y completar ta- } \\
\text { blas de sintesis. }\end{array}$ \\
\hline & $\begin{array}{l}\text { Rojas, P. (2001). „Pensamiento mé- } \\
\text { trico: Construcción del concepto } \\
\text { de medida». En: Rojas, P. (comp.). } \\
\text { Memorias Tercer Encuentro Co- } \\
\text { lombiano de Matemática Educa- } \\
\text { tiva (pp. 18-19). Bogotá: Gaia. }\end{array}$ & \\
\hline $\begin{array}{l}\text { PowerPoint sobre } \\
\text { pensamiento } \\
\text { geométrico. }\end{array}$ & $\begin{array}{l}\text { Contenido sobre pensamiento } \\
\text { geométrico: conceptualización, } \\
\text { etapas de su desarrollo y carac- } \\
\text { terísticas fundamentales de cada } \\
\text { etapa. }\end{array}$ & $\begin{array}{l}\text { Creación de casos escogiendo al- } \\
\text { guna etapa del desarrollo del pen- } \\
\text { samiento geométrico. }\end{array}$ \\
\hline $\begin{array}{l}\text { Apuntes sobre } \\
\text { nociones previas } \\
\text { al cálculo. }\end{array}$ & $\begin{array}{l}\text { Pruebas piagetanas. Eismann P. } \\
\text { (Universidad de Chile). Apuntes de } \\
\text { la Escuela de Psicología de la Uni- } \\
\text { versidad Católica de Chile (Hermo- } \\
\text { silla, M., asignatura PSD-149). }\end{array}$ & $\begin{array}{l}\text { Elaboración de PPT para exponer } \\
\text { en clases sobre alguna de las no- } \\
\text { ciones previas al cálculo escogida } \\
\text { (conservación, clasificación, seria- } \\
\text { ción y transitividad). }\end{array}$ \\
\hline
\end{tabular}




\begin{tabular}{|c|c|c|}
\hline Tipo de recurso & Descripción del recurso & $\begin{array}{l}\text { Actividad de aprendizaje a } \\
\text { desarrollar en preclase }\end{array}$ \\
\hline $\begin{array}{l}\text { PowerPoint sobre } \\
\text { teorias del apren- } \\
\text { dizaje matemático. }\end{array}$ & $\begin{array}{l}\text { Teorías del aprendizaje matemáti- } \\
\text { co desde el enfoque conductista, } \\
\text { constructivista y cognitivista. }\end{array}$ & $\begin{array}{l}\text { Elaboración de un cuadro compa- } \\
\text { rativo de las teorías abordadas. }\end{array}$ \\
\hline \multirow[t]{2}{*}{$\begin{array}{l}\text { Artículo y Power- } \\
\text { Point sobre } \\
\text { aprendizaje ma- } \\
\text { temático desde } \\
\text { las Neurociencias }\end{array}$} & $\begin{array}{l}\text { Jacubovich, S. (2006). "Modelos } \\
\text { actuales del procesamiento del nú- } \\
\text { mero y el cálculo". Revista Argen- } \\
\text { tina de Neuropsicología, 7: 21-31. }\end{array}$ & $\begin{array}{l}\text { Creación de una pregunta infe- } \\
\text { rencial a partir de los recursos } \\
\text { estudiados, con su respectiva res- } \\
\text { puesta. }\end{array}$ \\
\hline & $\begin{array}{l}\text { PPT con ideas fuerzas desde libro } \\
\text { especializado. Dehaene, S. (2016). } \\
\text { El cerebro matemático. Buenos Ai- } \\
\text { res: Siglo veintiuno. }\end{array}$ & \\
\hline
\end{tabular}

Fuente: Elaboración propia.

2. Sesión presencial: las aprendices llevaron las actividades resueltas a la clase presencial. Como primer paso, en cada sesión presencial se realizó una introducción, donde se contextualizó la clase y se activaron conocimientos previos sobre la clase anterior (5 minutos). Un segundo paso consideró la realización de preguntas aclaratorias, sugerido en Fodnes (2016) y replicadas en Espinoza y Araya (en prensa). Las preguntas son sobre las actividades preclase, y fueron mayormente respondidas entre las mismas aprendices y mediadas por la docente (10 minutos). En un tercer paso, se efectuaron las actividades de desarrollo (60 minutos) dando las instrucciones, y considerando las siguientes modalidades posibles: debate intergrupo sobre los insumos elaborados en la preclase, reestructuración de respuestas o insumo preclase según socialización intergrupo en la clase, elaboración de mapa conceptual con el curso a partir de los aportes de cada grupo, socialización y reestructuración de cuadros comparativos, exposición formativa de una temática y preguntas asociadas, entre otros. En esta parte de desarrollo, el rol activo fue dado a las estudiantes, mientras la docente tomaba más bien un rol mediador, efectuando preguntas, moderando el tiempo y turnos de trabajo. Como último paso, se efectuaba el cierre que tomaba unos 15 minutos, orientado a efectuar la síntesis de la clase y actividades, preguntas aclaratorias, y posibles aplicaciones de lo aprendido al contexto laboral en un futuro como educadoras diferenciales. 


\section{Resultados}

Los resultados obtenidos a partir de la ejecución del modelo de clase invertida pueden, por una parte, valorarse desde el rendimiento académico de las estudiantes y, por otra, desde la satisfacción de las estudiantes respecto de su aprendizaje a lo largo de la unidad 1 de la asignatura.

\subsection{Resultados considerando el rendimiento académico}

Sobre la valoración desde el rendimiento académico de las estudiantes, cabe destacar que en la primera clase se presentó el programa de la asignatura, donde se definían las evaluaciones sumativas en una escala de notas del 1 al 7. La unidad 1, "Desarrollo y Metodología de la Enseñanza de las Matemáticas», en la cual se llevó a cabo el diseño didáctico, tenía un peso de $30 \%$ en la nota final de la asignatura, del cual el 10 \% correspondía a talleres y el $20 \%$ a una evaluación escrita individual al finalizar la unidad. Es importante señalar que los pesos de las evaluaciones y las situaciones evaluativas se encontraban definidos previamente en el programa de la asignatura.

En relación con los talleres $(10 \%$ del peso en la nota final de la asignatura), los insumos elaborados grupalmente en la sesión autónoma o preclase se consideraron como tal. Del total de estudiantes (36), un $20 \%$ (7 estudiantes) presentaron un rendimiento muy bueno (calificación entre 6.0 y 7.0). Un 58 \% (21 estudiantes) presentaron un rendimiento bueno (calificación entre 5.0 y 5.9). Un $14 \%$ (5 estudiantes) presentaron rendimiento suficiente (calificación entre 4.0 y 4.9), debido a la no entrega de al menos una de las actividades de preclase. Por último, un 8 \% (3 estudiantes) presentaron rendimiento insuficiente (calificación inferior a 4.0) debido a la no entrega de dos o más actividades autónomas o talleres.

Respecto de la evaluación final sumativa (20 \% del peso en la nota final de la asignatura), como se señaló anteriormente, se efectuó una evaluación escrita individual. Para la realización de esta instancia, primeramente se hizo una clase preevaluación para aclarar dudas a modo de tutoría con pares y la docente. Días después, se efectuó la evaluación en una hora y media (un bloque de clase). Posteriormente, se entregó la evaluación a 
cada estudiante y se efectuó retroalimentación para aclaración dudas e identificación de errores cometidos como oportunidad de aprendizaje para su reestructuración. La evaluación consideraba los contenidos abordados tanto en preclase como clase, y las modalidades de preguntas o situaciones eran similares a las tratadas durante el proceso de aprendizaje. Del total de estudiantes, un $56 \%$ (20 estudiantes) aprobaron la evaluación sin mayor dificultad. Un $36 \%$ (13 estudiantes) no aprobaron la evaluación, aunque su calificación era recuperable. Por último, un $8 \%$ (3 estudiantes) presentaron rendimiento insuficiente y no recuperable en la evaluación escrita.

\subsection{Resultados considerando la valoración de las estudiantes}

En relación con la valoración de las estudiantes sobre su propio aprendizaje y su compromiso con el sistema de la clase durante la unidad 1, se recogió información por medio de una pauta de autoevaluación que constó de 14 indicadores agrupados en 4 dimensiones o áreas. Se consideraron como dimensiones: autovaloración del trabajo personal o autónomo en la preclase; autovaloración de la participación activa en la clase presencial; rol dentro del trabajo en equipo, y autopercepción del logro de los aprendizajes en la unidad 1. Al aplicar el instrumento, se explicó su propósito, las dimensiones antes mencionadas, así como los niveles de desempeño a registrar por indicador o reactivo (destacado, competente, básico e insatisfactorio). Por último, las estudiantes registraron los aprendizajes más significativos alcanzados, fortalezas y debilidades en su proceso de aprendizaje. Los resultados de la autoevaluación por dimensión se encuentran en la figura 1.

Sobre la dimensión de autovaloración del trabajo personal o autónomo en la preclase, las aprendices declararon que participaron activamente en el desarrollo de las actividades autónomas o de preclase (85,4\% de acuerdo), que llevaron a la clase presencial el taller resuelto en su totalidad ( $83,3 \%$ de acuerdo) y que previo a la clase leyeron comprensivamente el material digital $(77,1 \%$ de acuerdo). Se puede apreciar que, aunque declaran leer el material en menor medida, declaran mayormente que participan en la ejecución de la actividad autónoma y que asisten a la clase con el insumo realizado. 
Figura 1. Autoevaluación de las estudiantes sobre su desempeño durante la experiencia didáctica.

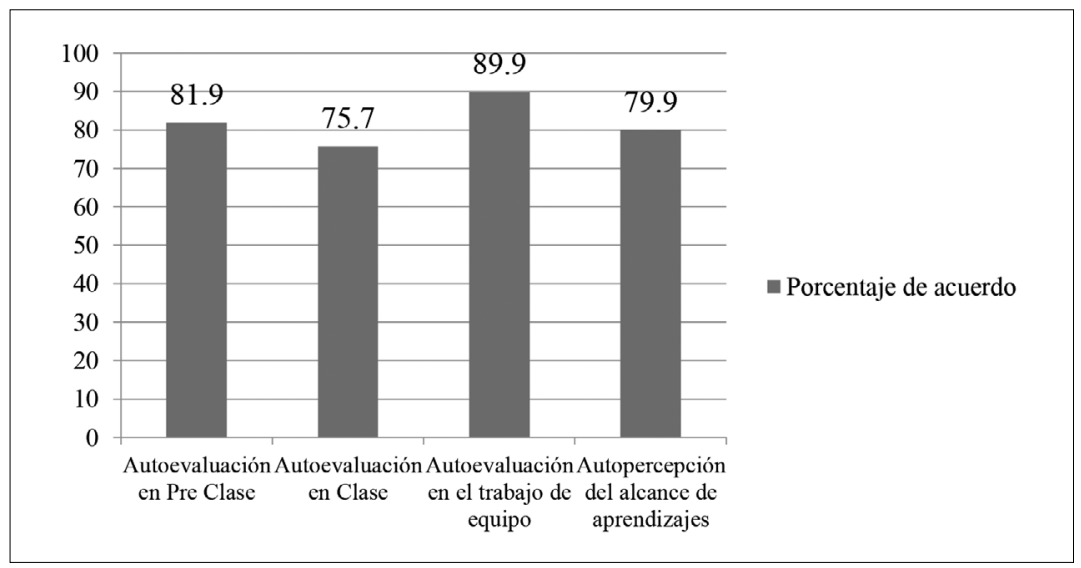

Fuente: Elaboración propia.

En relación con la dimensión de valoración de la participación activa en la clase presencial, las estudiantes declararon haber reestructurado y enriquecido sus aprendizajes en el proceso de clase presencial (88,2 \% de acuerdo), y que demostraron una postura analítica y reflexiva en la clase (72,2 \% de acuerdo). Sin embargo, en menor medida declararon que durante la clase propusieron y respondieron preguntas que les permitieran profundizar sus aprendizajes (66,7 \% de acuerdo).

Respecto de la dimensión rol dentro del trabajo en equipo, las estudiantes señalaron que se incluyeron en el trabajo en equipo, mostrando interés en ello (93,1\% de acuerdo). Asimismo, declararon que colaboraron con la discusión, retroalimentación y propuestas de mejora de los insumos elaborados en el equipo (87,5 \% de acuerdo). Por último, que tuvieron una participación activa en el equipo de trabajo durante la preclase y la clase presencial (86,1\% de acuerdo).

Por último, y asociado a la dimensión autopercepción del logro de los aprendizajes en la unidad 1, las estudiantes mostraron mayor acuerdo en el alcance de la comprensión y diferenciación de las nociones previas al cálculo (83,3\% de acuerdo). Asimismo, en el alcance de la comprensión de la evolución del pensamiento matemático en los niños/as, según las propuestas de etapas evolutivas de diversos autores $(80,6 \%$ de acuerdo). 
Además, manifestaron conocer y diferenciar los diferentes tipos de aprendizaje matemático: aditivo, métrico, geométrico, entre otros (79,2 \% de acuerdo). Por último, señalaron en menor medida alcanzar aprendizajes asociados a comprender los aportes de las ciencias cognitivas y neurociencias sobre el aprendizaje matemático $(76,4 \%$ de acuerdo).

\section{Discusión y conclusiones}

La ejecución de la clase invertida o flipped classroom, como se ha señalado en la literatura (Morgan et al., 2015), ha sido en general positiva también en la innovación didáctica aquí descrita. Los mayores insumos obtenidos para la valoración de la innovación fueron obtenidos por medio de procesos de evaluación permanente, y que consideraron tanto a las estudiantes como a la docente como agente evaluador. La literatura señala que los procesos evaluativos son débiles cuando se implementa la clase invertida (Karabulut-Ilgu, Jaramillo y Jahren, 2018), pues suele dirigirse a valorar la eficacia del diseño didáctico implementado y no considera otros aspectos como el real aprendizaje alcanzado por el estudiantado. Es por ello que en esta innovación didáctica se consideró de manera estructurada e intencionada, tomando en cuenta los elementos mencionados.

A partir de los insumos recogidos y sistematizados en los resultados, se detectan fortalezas y debilidades en la implementación del diseño didáctico. Como fortaleza se destaca que la mayoría de las estudiantes lograron aprobar la asignatura y manifestaron una alta valoración del logro de los aprendizajes. Además, se valoró positivamente la implementación de este modelo pedagógico de clase invertida y el trabajo en equipo, y se constató que era coherente con la literatura (Kong, 2014). Por otro lado, como debilidad se detectó una diferencia en el rendimiento de las estudiantes en la evaluación de talleres o actividades autónomas, y la evaluación individual final de la asignatura. Las estudiantes que participaron sistemáticamente en preclases y clases fueron quienes obtuvieron mejor rendimiento general en la unidad. No obstante, las estudiantes tendieron a rendir mejor en las actividades autónomas grupales que en la evaluación individual final, aunque ambas consideraron los 
mismos contenidos y la misma modalidad de preguntas o situaciones. Esta discrepancia puede deberse a que, en equipo, las estudiantes se apoyan entre sí, logrando un producto o insumo mejorado gracias a la intervención de varias personas coordinadas. Mientras que, en la evaluación individual, se demuestra cuánto de los aprendizajes alcanzados en el proceso, por medio de las actividades autónomas, efectivamente persisten en cada estudiante de manera individual.

Como conclusiones, primeramente es importante resaltar la importancia de aplicar diseños didácticos que se basen en la evidencia o la literatura, tal como lo es la clase invertida. En segundo lugar, es trascendental que las actividades autónomas de preclase tengan un peso en la nota final de la asignatura, pues son demostraciones de los aprendizajes que se alcanzan durante la trayectoria formativa considerada en la unidad. Además, porque se da peso al proceso de aprendizaje efectuado en el tiempo personal, estipulado en los programas de aprendizaje que consideran Sistema de Créditos Transferibles (SCT-Chile). Para finalizar, cabe destacar que el modelo pedagógico facilitó en esta experiencia el aprendizaje de contenidos complejos, teóricos y abstractos, y se pudo solventar el problema que originó la implementación del diseño didáctico. Como proyección de la innovación, se sugiere la implementación de flipped classroom, tanto en otras carreras de pregrado como en la formación técnico-profesional de nivel superior por su efectividad.

\section{Referencias bibliográficas}

Basal, A. (2015). «The implementation of a flipped classroom in foreign language teaching». Turkish Online Journal of Distance Education, 16(4): 28-37.

Blair, E.; Maharaj, C.; Primus, S. (2015). «Performance and perception in the flipped classroom». Education and information Technologies, 21(6): 1465-1482.

Deyasi, A.; Bhattacharyya, S.; Debnath, P.; Mukherjee, S.; Kumar, A. (2019). «Effective utilization of digital resources for undergraduate technical education through flipped learning for performance improvement». Proceedings of International Conference on Digital Pedagogies (ICDP, 2019). Nueva Dehli (India). 
Espinoza, L.; Araya, A. (2019). "Clase invertida y aprendizaje cooperativo en posgrado: Una experiencia en Chile». EDUCERE: Revista Venezolana de Educación, 75: 477-486.

Foldnes, N. (2016). «The flipped classroom and cooperative learning: Evidence from a randomised experiment». Active Learning in Higher Education, 17(1): 39-49.

Jensen, J.; Kummer, T.; Godoy, P. (2014). «Improvements from a flipped classroom may simply be the fruits of active learning». Life Sciences Education, 14: 1-12.

Johnson, D.; Johnson, R. (2014). «Cooperative Learning in 21st Century». Anales de Psicología, 30(3): 841-851.

Karabulut-Ilgu, A.; Jaramillo, N.; Jahren, C. (2018). «A systematic review of research on the flipped learning method in engineering education». British Journal of Educational Technology, 49(3): 398-411.

Kong, S. (2014). «Developing Information literacy and critical thinking skills through domain knowledge learning in digital classrooms: An experience of practicing flipped classroom strategy». Computers \& Education, 78: 160-173.

Menegaz, J.; Dias, G.; Trindade, R.; Leal, S.; Martins, N. (2018). «Flipped classroom in teaching nursing management: Experience report». Escola Anna Nery, 22(3): 1-7.

Mok, H. N. (2014). «Teaching tip: The flipped classroom». Journal of Information Systems Education, 25(1): 7-11.

Morgan, H.; McLean, K.; Chapman, C.; Fitzgerald, J.; Yousuf, A.; Hammoud, M. (2015). «The flipped classroom for medical students». The Clinical Teacher, 12: 155-160.

Peterson, D. (2016). «The flipped classroom improves student achievement and course satisfaction in a statistics course: A quasi-experimental study». Teaching of Psychology, 43(1): 10-15. 

1. La innovación educativa en el contexto de la

Educación Superior Técnico-Profesional.

Claudio Maregatti Solano, María luisa Arancibia Muñoz, Rosa Eliana Romero Alonso

1. Introducción

2. La transformación de las instituciones de

Educación Superior Técnico-Profesional

3. De las prácticas docentes al saber académico

4. El aporte al conocimiento científico sobre la

Educación Superior Técnico-Profesional . . . . . . . . . 15

5. Referencias bibliográficas .................. . 21

2. Caracterización de estudiantes de Educación Técnico-Profesional respecto a su Educación Secundaria: reflexiones para la Educación Superior

Javier Torres-Vallejos, Juan Ignacio Venegas Muggli, Simón Mundaca Toledo, Juan Carlos Oyanedel Sepúlveda

1. Introducción . . . . . . . . . . . . . . . . . . . 26

2. Metodología ......................... . 30

2.1 Muestra . . . . . . . . . . . . . . . . . . . . . 30

2.2 Variables . . . . . . . . . . . . . . . . . . . . . 32

2.3 Tratamiento de bases de datos . . . . . . . . . . . . 32

2.4 Análisis de datos..................... 33

3. Resultados ........................... 33

3.1 Características educativas y académicas en

Educación Superior ..................... . 34

3.2 Características educativas y académicas del establecimiento secundario de egreso de Enseñanza Media . . . . . . . . . . . . . . . . . . . . 36

4. Discusión y conclusiones. . . . . . . . . . . . . . . 37

5. Referencias bibliográficas ................. . . 39 
3. Aportes hacia la construcción de un perfil docente para el primer año: la importancia del vínculo empático, el valor académico y el trabajo colaborativo

Carlos Alberto Acevedo Cossio

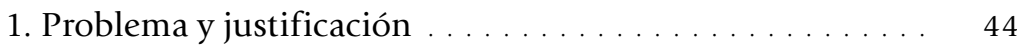

2. Marco teórico ....................... 45

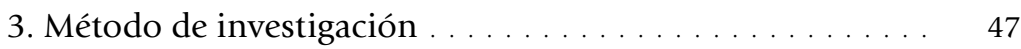

3.1 Diseño. . . . . . . . . . . . . . . . . . . 47

3.2 Participantes...................... 47

3.3 Técnicas e instrumentos de recolección de datos. . . . 48

3.4 Análisis de datos. . . . . . . . . . . . . . . . . . . . . 48

4. Resultados ... . . . . . . . . . . . . . . . . . . . . . . 49

4.1 Análisis de las entrevistas realizadas . . . . . . . . . 49

4.2 Análisis de cuestionarios . . . . . . . . . . . . . 50

4.2.1 Cuestionario de Estilo y Eficacia del Liderazgo . . 50

4.2.2 Cuestionario de Involucramiento Académico . . . 51

4.2.3 Índice de Reactividad Interpersonal . . . . . . . . . 52

5. Conclusiones y discusión ................. . 53

6. Referencias bibliográficas .................. 55

4. Mentoría docente para instalar estrategias de resolución de problemas en matemáticas iniciales

Cristian Iván Ramos Arrepol, Carlos Alberto Acevedo

Cossio

1. Problema y justificación . . . . . . . . . . . . . . . . 58

2. Marco teórico . . . . . . . . . . . . . . . . . . . . . . . 59

2.1 Trabajo colaborativo . . . . . . . . . . . . . . . . . 59

2.2 Mentorías ......................... 60

3. Método de investigación .................... 61

3.1 El perfil de entrada del docente . . . . . . . . . . . 62

3.2 Reclutamiento docente. . . . . . . . . . . . . 62

3.3 La caminata de aula . . . . . . . . . . . . . . . . . . 63

3.3.1 El primer paso . . . . . . . . . . . . . . . . 63

3.3 .2 Segunda ronda . . . . . . . . . . . . . . . . . . 64

3.3.3 Ronda final. . . . . . . . . . . . . . . . . . 64

3.4 Abordaje de situaciones críticas . . . . . . . . . . . . 64

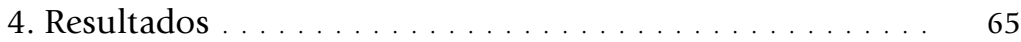

5. Conclusiones y discusión. . . . . . . . . . . . . . . . 69

6. Referencias bibliográficas ................... 70 
5. Clase invertida para la formación inicial de educadoras diferenciales sobre aprendizaje matemático

Laura Marjorie Espinoza Pastén

1. Problema y justificación . . . . . . . . . . . . . . . . . . 74

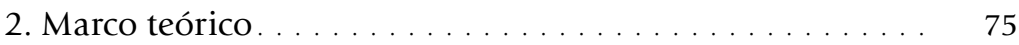

3. Proceso de la innovación. . . . . . . . . . . . . . . 76

3.1 Contexto ........................ 76

3.2 Diseño didáctico . . . . . . . . . . . . . . . 77

4. Resultados ......................... . 80

4.1 Resultados considerando el rendimiento académico. . 80

4.2 Resultados considerando la valoración de las estudiantes $\quad 81$

5. Discusión y conclusiones . . . . . . . . . . . . . . . 83

6. Referencias bibliográficas ................. 84

6. ¿Cómo medir el aprendizaje en innovación? Análisis factorial confirmatorio del Innovator's Behavior Questionnaire (i)BQ en universitarios chilenos. . . . . . 87 Jorge Maluenda Albornoz, Pedro Lledó Aninat

1. Problema y justificación . . . . . . . . . . . . . . . . . . . . . 89

2. Marco teórico . . . . . . . . . . . . . . . . . . . . . 90

3. Método............................. 92

4. Resultados ......................... 93

5. Conclusiones y discusión. . . . . . . . . . . . . . . . . 96

6. Referencias bibliográficas ................. 98

7. Programa de inclusión para actores de Educación Superior Técnico-Profesional . . . . . . . . . . . . . . . . 101

Marcela Pérez Poquet, Paulina Muñoz Villalobos, Victoria Aravena Rivas

1. Problema y justificación del estudio . . . . . . . . . . . . 102

2. Marco teórico . . . . . . . . . . . . . . . . . . . . . . 105

3. Metodología de investigación ................ 108

3.1 Descripción del contexto . . . . . . . . . . . . . . . 108

3.2 Participantes. . . . . . . . . . . . . . . . . . . . 109

3.3 Instrumentos y procedimientos . . . . . . . . . . . . . . 109

4. Resultados . . . . . . . . . . . . . . . . . . . . . . . 111

5. Conclusiones y discusión. . . . . . . . . . . . . . . . . . 113

6. Referencias bibliográficas ................. 113

Los autores. . . . . . . . . . . . . . . . . . . . . . . . . ${ }^{115}$ 